\title{
Política Nacional de Humanização e formação dos profissionais de saúde: revisão integrativa
}

\author{
National Policy of Humanization and education of health care professionals: integrative review
}

Política Nacional de Humanización y formación de los profesionales de salud: revisión integrativa

\section{Guilherme Correa Barbosa', Silmara Meneguim', Silvana Andréa Molina Lima', Vania Moreno'}

' Universidade Estadual Paulista, Faculdade de Medicina de Botucatu, Departamento de Enfermagem. Botucatu-SP, Brasil.

\author{
Submissão: 30-06-2011 Aprovação: 22-01-2013
}

\section{RESUMO}

A Política Nacional de Humanização tem como objetivo provocar inovações na produção de saúde, gestão e no cuidado, com ênfase na educação permanente dos trabalhadores do Sistema Único de Saúde e na formação dos acadêmicos da área de saúde. Buscou-se conhecer, através de uma revisão integrativa, a produção científica sobre a Política Nacional de Humanização e a educação de trabalhadores e alunos da área da saúde, no período de 2002 a 2010. Dez artigos foram analisados na vertente temática e emergiram três eixos: humanização e o cuidado aos usuários, humanização e o processo de trabalho, humanização e a formação. Os artigos apontam a necessidade de superação da concepção biologicista e valorização dos aspectos culturais dos usuários. O processo de trabalho é marcado pela desvalorização dos trabalhadores e por usuários destituídos de seus direitos. A formação dos profissionais de saúde está pautada em serviços de saúde em que prevalece a padronização de condutas o que dificulta atitudes inovadoras.

Descritores: Humanização da Assistência; Política de Saúde; Educação em Enfermagem.

\begin{abstract}
The National Policy of Humanization aims at innovations in health production, management and care with emphasis on permanent education for workers in the Unified Public Health System and training of university students in the health care field. This study aimed to know, through an integrative review of the literature, the scientific production about the National Policy of Humanization and education of health care professionals, from 2002 to 2010. Ten articles were analyzed in thematic strand through three axes: humanization and users caring, humanization and the work process, humanization and training. The articles point to the need to overcome the biological conception, valuing cultural aspects of users. The work process is marked by the devaluation of workers and by users deprived of their rights. The training of health professionals is grounded in health services where the prevailing standards are practices that hinder innovative attitudes.
\end{abstract}

Key words: Humanization of Assistance; Health Policy; Education Nursing.

\section{RESUMEN}

La Política Nacional de Humanización tiene como objetivo provocar innovaciones en la producción de salud, en la gerencia y en el cuidado con énfasis en la educación permanente de los trabajadores del Sistema Único de Salud y en la formación de los académicos del área de salud. En este estudio se procuró conocer, a través de una revisión integrativa, la producción científica sobre la Política Nacional de Humanización y la educación, en el período de 2002 a 2010 . Diez artículos fueron analizados en un enfoque temático a través de tres ejes: humanización y atención a los usuarios, humanización y el proceso de trabajo, y humanización y formación. Los artículos apuntan a la necesidad de superar la concepción biologicista, valorando los aspectos culturales de los usuarios. El proceso de trabajo se caracteriza por la devaluación de los trabajadores y por usuarios privados de sus derechos. La formación de los profesionales de la salud se basa en servicios de salud, donde prevalecen las prácticas habituales vigentes que impiden actitudes innovadoras.

Palabras clave: Humanización de la Atención; Política de Salud; Educación en Enfermería. 


\section{INTRODUÇÃO}

No Brasil, em 1999 foi instituído o Programa Nacional de Humanização da Assistência Hospitalar que teve como justificativa: agregar a eficiência técnica e científica a uma postura ética que respeitasse a singularidade das necessidades do usuário e do profissional, aceitando os limites de cada um e a convivência com o desconhecido e o imprevisível ${ }^{(1)}$.

Em 2003, o Ministério da Saúde implanta a Política Nacional de Humanização da Atenção e Gestão do Sistema Único de Saúde, que tem como princípios teóricos metodológicos: a transversalidade, a inseparabilidade entre atenção e gestão e o protagonismo dos sujeitos e coletivos ${ }^{(2)}$. Amplia-se, assim, o campo da assistência hospitalar para todos os serviços de atenção a saúde.

A humanização se configura como uma aposta ética, estética e política. Ética, pois implica que usuários, gestores e trabalhadores estejam comprometidos com a melhoria do cuidado, estética porque permite um processo criativo e sensível da produção da saúde por sujeitos autônomos e protagonistas de um processo coletivo. Político refere-se à organização social e institucional, onde se espera que haja solidariedade dos vínculos estabelecidos, dos direitos dos usuários e da participação coletiva do processo de gestão ${ }^{(3)}$.

$\mathrm{O}$ ensino torna-se um aliado nas mudanças que devem ocorrer em dois movimentos distintos: o primeiro, com os profissionais de saúde onde a formação acontece via educação permanente que tem como ponto de partida o cotidiano do trabalho. E, no segundo momento, parte da ruptura do ensino de graduação em saúde pautado em procedimentos técnicos e de evolução dos quadros clínicos para um território onde a "educação em ato" ocorre através de práticas cuidadoras, com inovação e centrada no diálogo com o usuário e equipe, buscando criar os nexos necessários entre saúde, educação e trabalho(4).

\section{MÉTODO}

A revisão integrativa foi escolhida porque corresponde a um método de pesquisa que viabiliza análise de pesquisas científicas de modo sistemático e amplo, favorecendo a caracterização do conhecimento produzido sobre humanização e educação. Permitindo ainda revelar lacunas existentes do conhecimento sobre a temática estudada(5).

Para a realização da presente revisão, seis etapas foram percorridas: estabelecimento do problema de revisão; seleção da amostra; categorização dos estudos; análise dos resultados; apresentação e discussão dos resultados; e por fim, apresentação da revisão(6).

O estudo foi direcionado pelo seguinte questionamento: Qual a contribuição das investigações científicas publicadas nos periódicos, durante o período de 2002 a 2010 sobre a Política Nacional de Humanização $(\mathrm{PNH})$ e a educação de trabalhadores e alunos da área de saúde?

Para identificar os estudos publicados sobre a Política Nacional de Humanização e educação, foi efetuada uma busca on-line nas bases de dados da Literatura Latino-Americana e do Caribe em Ciências da Saúde (LILACS) no sítio da Biblioteca Virtual em Saúde (BVS), durante os anos 2002 a 2010. Utilizou como descritores: humanização e educação. Esta busca se deu no decorrer do mês de fevereiro de 2011.

Foram identificados, no universo pesquisado, 112 documentos. Considerou-se como critérios de inclusão para essa revisão: artigos em periódicos nacionais, indexados no banco de dados selecionados, que abordassem a $\mathrm{PNH}$ e o processo de formação de trabalhadores e discentes dos cursos de graduação na área de saúde. Foram excluídos: teses, dissertações, artigos em revistas latino-americanas e de outros países e artigos que não se relacionavam à temática estudada. Identificou-se uma amostra de dez artigos, que foram, então, analisados.

Elaborou-se como instrumento de coleta de dados um formulário contendo os seguintes dados: Nome do periódico, ano de publicação, título do artigo, nome dos autores, método da investigação. Em relação aos autores: profissão dos autores, instituição e região do país que foi desenvolvido o estudo.

Os artigos relacionados à temática estudada são apresentados no Quadro 1.

Dos dez artigos, cinco eram de pesquisa (um com abordagem qualitativa e um exploratório quantitativo), três eram ensaios, e dois eram relatos de experiência sobre a implantação da PNH nos serviços de saúde.

Quanto à autoria, predominaram os enfermeiros, porém há autores médicos, assistentes sociais, antropólogos e psicólogos. Três artigos estão vinculados a Programa de Pós-Graduação. Os autores estão vinculados a universidades públicas, estaduais e federais. Um dos artigos foi conduzido em universidade de caráter privada, mas com função social. Os autores são predominantemente da Região Sul e Sudeste; em um artigo os autores eram da região Nordeste do país.

Nos periódicos em que foram publicados os artigos, seis têm como objetivo difundir os conhecimentos advindos da área da saúde coletiva, dois são vinculados à enfermagem e dois promovem a divulgação da $\mathrm{PNH}$ na área da saúde.

A leitura exaustiva da íntegra dos artigos permitiu a construção dos seguintes eixos temáticos: humanização e o cuidado com os usuários, humanização e o processo de trabalho, humanização e a formação.

\section{RESULTADOS E DISCUSSÃO}

\section{A. Humanização e o cuidado com os usuários}

Para a construção de uma nova forma de cuidado com os usuários dos serviços de saúde pautados na humanização, leva-se em consideração que o usuário deva ter uma abordagem integral e humana. Portanto, devem ser respeitados os seus saberes que são ligados a sua cultura e que dão sustentação a sua forma de perceber seu processo de adoecimento ${ }^{(7)}$.

Nos espaços de saúde, o encontro físico que acontece em um consultório onde o usuário busca alívio para seus problemas de saúde também deve ser entendido como um encontro de culturas(7).

A cultura, presente na vida das pessoas que procuram os serviços de saúde, deve ser valorizada, não se restringindo 


\begin{tabular}{|c|c|c|c|}
\hline Ano & Revista & Autor & Titulo \\
\hline 2002 & Interface & Oliveira FA & $\begin{array}{l}\text { Antropologia nos serviços de saúde: integralidade, cultura e } \\
\text { comunicação. }\end{array}$ \\
\hline 2005 & Ciência \& saúde coletiva & Hotimsky SN, Schraiber LB & Humanização no contexto da obstetrícia \\
\hline 2006 & Rev Esc Enferm USP & $\begin{array}{l}\text { Casate JC, } \\
\text { Corrêa AK }\end{array}$ & $\begin{array}{l}\text { Vivências de alunos de enfermagem em estágio curricular hospitalar: } \\
\text { subsídios para refletir sobre a humanização em saúde }\end{array}$ \\
\hline 2006 & Cadernos de Saúde Coletiva & $\begin{array}{l}\text { Barros MEB, } \\
\text { Mori ME, } \\
\text { Bastos SS }\end{array}$ & $\begin{array}{l}\text { O desafio da Política Nacional de Humanização nos processos } \\
\text { de trabalho: o instrumento "Programa de Formação em saúde e } \\
\text { trabalho". }\end{array}$ \\
\hline 2009 & Interface & $\begin{array}{l}\text { Ceccim RB, } \\
\text { Merhy ED }\end{array}$ & $\begin{array}{l}\text { O agir micropolítico e pedagógico intenso: a humanização entre } \\
\text { laços e perspectivas }\end{array}$ \\
\hline 2009 & Interface & $\begin{array}{l}\text { Gomes AMA, } \\
\text { Sampaio JJC, Carvalho MGB, } \\
\text { Nations MK, } \\
\text { Alves MSCF }\end{array}$ & $\begin{array}{l}\text { Código dos direitos e deveres da pessoa hospitalizada no SUS: o } \\
\text { cotidiano hospitalar na roda de conversa }\end{array}$ \\
\hline 2009 & Einstein & $\begin{array}{l}\text { Almeida DV, } \\
\text { Chaves ED }\end{array}$ & $\begin{array}{l}\text { O ensino da humanização nos currículos de graduação em } \\
\text { enfermagem }\end{array}$ \\
\hline 2009 & Interface & $\begin{array}{l}\text { Mello VC, } \\
\text { Bottega CG }\end{array}$ & $\begin{array}{l}\text { A prática pedagógica de formação da Política Nacional de } \\
\text { Humanização }(\mathrm{PNH})\end{array}$ \\
\hline 2009 & O mundo da Saúde & Waldow VR & $\begin{array}{l}\text { Reflexões sobre Educação em Enfermagem: ênfase em um ensino } \\
\text { centrado no cuidado }\end{array}$ \\
\hline 2010 & Rev Bras Enferm & Corbellini VL et al & Nexos e desafios na formação profissional do enfermeiro \\
\hline
\end{tabular}

Quadro 1 - Publicações encontradas no LILACS sobre a produção científica da Política Nacional de Humanização e a formação dos profissionais e acadêmicos de saúde, no período de 2002 a 2010.

apenas ao atendimento queixa-sintoma-tratamento, o que diminui a compreensão das subjetividades envolvidas na construção de trocas solidárias e comprometidas ${ }^{(7,3)}$.

A Política de Humanização também traz, como desafio a ser superado, a fragmentação existente nas atividades programáticas, qual seja: humanização da assistência hospitalar, do parto, do nascimento, do morrer, entre outros ${ }^{(8)}$.

Cabe aos profissionais romper com a lógica presente na assistência, marcada pela prática de atender apenas a queixas, passando a traçar possibilidades de efetivo encontro entre trabalhador-usuário e trabalhador-trabalhador, em que as pessoas não sejam vistas como um diagnóstico de doença ${ }^{(8)}$.

Os encontros devem ser marcados pela singularidade e pela subjetividade dos sujeitos envolvidos e à medida que se transformam o cotidiano dos serviços permitem que as práticas pedagógicas se façam presentes nos saberes e fazeres ${ }^{(8,4)}$.

\section{B. Humanização e o processo de trabalho}

Para implantar a Política de Humanização, um dos desafios é enfrentar as condições de trabalho a que estão submetidos os trabalhadores: desvalorização, precarização e baixo investimento em educação permanente, um modelo de gestão centralizado e vertical que impossibilita os trabalhadores de se apropriar de seu próprio processo de trabalho ${ }^{(9)}$.

Uma experiência é o Programa de Formação em Saúde e Trabalho (PFST) que aponta para a necessidade de instrumentalizar os trabalhadores em um primeiro momento através de curso e, em um segundo momento, através de estudo de campo, encontros para a discussão de possíveis mudanças nas situações de trabalho ${ }^{(9)}$. Este processo em construção está pautado no dialogo-confrontação entre as ferramentas oferecidas pelos coordenadores do Programa e o cotidiano vivenciado pelos trabalhadores ${ }^{(9)}$.

A expectativa é a constituição de uma rede de trabalhadores preparados para analisar e intervir nos seus espaços de trabalho. A busca é pelo protagonismo dos trabalhadores, corresponsáveis, estabelecendo laços solidários, tecendo redes de cooperação e participando coletivamente do processo de gestão ${ }^{(9,3)}$.

A utilização de um Curso de Especialização em Humanização de Atenção e Gestão do SUS também foi uma ferramenta utilizada para formar profissionais no âmbito da Política Nacional de Humanização(10).

O curso-intervenção foi oferecido na modalidade à distância e permitiu agrupar trabalhadores de vários municípios e região do Estado do Rio Grande do Sul em encontros presenciais e em atividades de ensino à distância, visando formar apoiadores institucionais para a Política Nacional de Humanização ${ }^{(10)}$.

No curso-intervenção, o aluno/apoiador era convidado a propor uma intervenção no seu local de trabalho, cenário ideal para problematizar, disputar e construir um projeto de saúde voltado para sua realidade de trabalho. Portanto, o currículo foi um caminhar construído a partir das intervenções realizadas pelos alunos e compartilhadas com o coletivo do curso, por permitir uma variedade de experiências e produzir discussões a partir do vivido de cada aluno/apoiador ${ }^{(10)}$. 
As inovações podem acontecer em espaços como os hospitais que utilizam a rede de conversa, levando aos profissionais a oportunidade de discutir sobre atitudes e possibilidades dos usuários conhecerem seus direitos ${ }^{(11)}$.

A roda de conversa se constitui uma estratégia educativa cujo objetivo é permitir que os trabalhadores no primeiro momento repensem suas práticas rotineiras e proponham mudanças concretas, com o objetivo de garantir os direitos dos usuários $^{(11)}$.

Os artigos analisados permitem concluir que, para ocorrerem movimentos nos serviços de saúde em busca da implantação da Política Nacional de Humanização, há necessidade da formação de trabalhadores com possibilidade de produzir novos preceitos e novas maneiras de se conformar a vida e o trabalho. Porém, a produção de saberes está nos espaços de saúde e são construídos coletivamente ${ }^{(12)}$.

\section{Humanização e a formação}

Com a contribuição da etnografia, investigou-se a formação médica no contexto de um programa interdisciplinar e interdepartamental que visava discutir a saúde da mulher e a saúde reprodutiva ${ }^{(13)}$.

A partir da sala de aula e de visitas a serviços públicos de "assistência humanizada ao parto", pode-se constatar que a noção de humanização é polêmica e que se contrapõe à proposta ministerial, criando embates ${ }^{(13)}$.

O aluno apresenta dificuldade em lidar com o pluralismo de correntes que permeiam a assistência ao parto e, consequentemente, às intervenções possíveis, sendo uma prática dos serviços o uso de protocolos ${ }^{(13)}$.

Investigações realizadas na formação do enfermeiro pautaram-se nas Diretrizes Curriculares Nacionais do Curso de Graduação em Enfermagem como norteadoras das pesquisas e reflexões, que enfatizam a dimensão humanista do cuidado.

Alunos de graduação de uma universidade pública referem despreparo para uma atitude de escuta, envolvimento e acoIhimento com o sofrimento apresentado pelo paciente/usuário dos serviços. Prevalece o fazer técnico em detrimento da humanização das práticas ${ }^{(14)}$.

Os autores indicam a necessidade de articulação de conteúdos das ciências humanas com os conteúdos clínicos e que os espaços de formação possibilitem o compartilhamento de saberes entre serviços e instituição formadora, para produzirem novas relações entre professores, alunos e profissionais de saúde $e^{(14,7)}$.

Um estudo documental buscou conhecer os conteúdos sugestivos da Política Nacional de Humanização, nos programas das disciplinas que compõem a estrutura curricular de 13 Cursos de Graduação em Enfermagem e revela que, apesar de o conteúdo estar presente em todas as escolas pesquisadas, as tendências são ambíguas na maneira de apresentar o conteúdo de humanização, sendo ofertadas em disciplinas diversas $^{(15)}$.

Esse estudo destaca ainda que, apesar de a Política Nacional de Humanização estar contemplada de forma significativa nos currículos, nenhuma disciplina mencionou documento do Ministério em seu referencial bibliográfico ${ }^{(15)}$.
Uma pesquisa com enfermeiros graduados que foram entrevistados visando conhecer os nexos e desafios entre a formação e a prática profissional indicou entre as temáticas analisadas a humanização. Porém, segundo os entrevistados no cotidiano dos serviços, a prática é marcada pela dimensão técnica em detrimento da dimensão humanista ${ }^{(16)}$.

Os autores trazem como reflexão a importância de estratégias pedagógicas, como exemplo as metodologias ativas com a ampliação da participação do professor e alunos nos campos de prática e integrados às políticas publicas de saúde(16).

A reflexão sobre a educação em enfermagem indica caminhos necessários para o ensino centrado no cuidado e, entre os desafios a serem enfrentados, estão as instituições de saúde que mantêm uma posição conservadora e reducionista e atitudes fragmentadas onde o paciente é objeto de conhecimento ou de lucro. Predomina a padronização de condutas ${ }^{(17)}$. Por outro lado, o agir pedagógico deveria se pautar em uma prática reflexiva onde o aluno possa adquirir competência para lidar com situações únicas, incertas e conflituosas ${ }^{(17)}$. O ensino alicerçado no cuidado implica uma relação interpessoal, imprevisível, que não pode ser pré-estabelecida, pois é única e peculiar à pessoa humana e potencialmente criativa ${ }^{(17)}$.

O ensino de graduação nas áreas de saúde deve formar profissionais com capacidade de solucionar os problemas, mas também inventar problemas, pois é a invenção que traz a possibilidade do novo e que está sempre implicado na imprevisibilidade, presente nos serviços quando se propõem encontros, roda de conversa e cursos de formação pautados na experiência cotidiana do trabalhador ${ }^{(18,8-10)}$.

\section{CONCLUSÃO}

Os artigos indicam a importância da incorporação das ciências humanas no campo da saúde visando proporcionar a ampliação de saberes para melhor conhecer a população a que se atende em seu processo de adoecimento.

Com relação ao processo de trabalho os artigos indicam alguns desafios a serem enfrentados, quais sejam: trabalhadores desvalorizados em seu cotidiano institucional, precarizados e baixo investimento em educação permanente.

Há indícios que os cursos de especialização que tem como foco a intervenção nos serviços de saúde, com ênfase na rede de trabalhadores e rodas de conversa são possibilidades que se apresentam frente as dificuldades encontradas na construção de um projeto coletivo envolvendo trabalhadores, usuários e gestores.

A formação dos alunos de graduação em enfermagem e medicina encontra como obstáculo o pluralismo do termo humanização, sem que se valorize o documento do Ministério da Saúde sobre Politica Nacional de Humanização.

Nos serviços de saúde ainda se mantém a padronização de condutas e o fazer técnico como forma de organização dos serviços em detrimento ao acolhimento e integralidade do cuidado. Segundo a Política Nacional de Humanização a busca é formar profissionais que na sua atuação articulem ações de eficiência técnica e científica, postura ética, mas que respeite a necessidade e singularidade de cada usuário, 
sabendo que está convivência é imprevisível e é geradora de inovação nas práticas de saúde.

Os três eixos analisados por esta investigação a humanização e cuidado com os usuários, a humanização e o processo de trabalho e a humanização e a formação indicam possibilidades de novas investigações sobre a temática de forma a construir um arcabouço teórico-prático imprescindível para a implementação do Sistema Único de Saúde.

\section{REFERENCIAS}

1. Ministério da Saúde. Secretaria de Assistência a Saúde. Programa Nacional de Humanização da Assistência Hospitalar. Brasília, DF: O Ministério; 2001.

2. Heckert ALC, Passos E, Barros MEB. Um seminário dispositivo: a humanização do Sistema Único de Saúde (SUS) em debate. Interface Comun Saúde Educ 2009;13(sup1);493-502.

3. Ministério da Saúde. Secretaria de Atenção à Saúde. Núcleo Técnico da Política Nacional de Humanização. HumanizaSUS: documento base para gestores e trabalhadores do SUS. 3.ed. Brasília: Ministério da Saúde, 2006.

4. Pinheiro R, Ceccim RB. Experienciação, formação, cuidado e conhecimento em saúde: articulando concepções, percepções e sensações para efetivar o ensino da integralidade. In: Pinheiro R, Ceccim RB, Mattos RA, organizadores. Ensinar Saúde: a integralidade e o SUS nos cursos de graduação na área da saúde. Rio de Janeiro: IMS/IERJ-CEPESQ-ABRASCO; 2005. p.13-33.

5. Mendes KDS, Silveira RCCP, Galvão CM. Revisão integrativa: método de pesquisa para a incorporação de evidências na saúde e na enfermagem. Texto contexto Enferm 2008;17(4):758-64.

6. Ganong LH. Integrative reviews of nursing research. Res Nurs Health 1987;10(1):1-11.

7. Oliveira FA. Antropologia nos serviços de saúde. Interface - Comunic Saúde Educ 2002;6(10):63-74.

8. Ceccim RB, Merhy EE. Um agir micropolítico e pedagógico intenso: a humanização entre laços e perspectivas. Interface - Comunic Saúde Educ 2009;13(sup1);531-42.

9. Barros MEB, Mori ME, Bastos SS. O desafio da Política Nacional de Humanização nos processos de trabaIho: o instrumento "Programa de Formação em saúde e trabalho". Cad Saude Pub 2006;14(1):31-48.

10. Mello VC, Bottega CG. A prática pedagógica no processo de formação da Política Nacional de Humanização (PNH). Interface Comun Saúde Educ 2009;13(sup1);739-46.

11. Gomes AMA, Sampaio JJC, Carvalho MGB, Nations MK, Alves MSCF. Código dos direitos e deveres da pessoa hospitalizada no SUS: o cotidiano hospitalar na roda de conversa. Interface Comun Saúde Educ 2008;12(27);773-82.

12. Heckert ALC, Neves CAB. Modos de formar e modos de intervir: quando a formação se faz potencia de produção de coletivo. In: Ministério da Saúde. Secretaria de Atenção a Saúde. Política Nacional de Humanização: formação e intervenção. Brasília: O Ministério; 2010. p. 13-28.

13. Hotimsky SN, Schraiber LB. Humanização no contexto da formação em obstetrícia. Cienc Saude Col 2005;10(3):639-49.

14. Casate JC, Corrêa AK. Vivências de alunos de enfermagem em estágio hospitalar: subsídios para refletir sobre a humanização em saúde. Rev Esc Enferm USP 2006;40(3):321-8.

15. Almeida DV, Chaves EC. O ensino de humanização nos currículos de graduação em enfermagem. Einstein 2009;7(3):271-8.

16. Corbellini VL, Santos BRL, Ojeda BS, Gerhart LM, Eidt $\mathrm{OR}$, Stein SC, et al. Nexos e desafios na formação profissional do enfermeiro. Rev Bras Enferm 2010;63(4):555-60.

17. Waldow VR. Reflexões sobre Educação em Enfermagem: ênfase em um ensino centrado no cuidado. Mundo Saúde 2009;33(2):182-88.

18. Kastrup V. Políticas cognitivas na formação do professor e o problema do devir-mestre. Educ Soc 2005; 26(93):1273-1288. 